\title{
Application of result of atoms and centrosymmetric cubic space groups for sharpening of Patterson function
}

\author{
P. S. Yuen \\ 237 Des Voeux Road West, 5th Floor, Hong Kong \\ puisumyuen@netvigator.com
}

$\mid \mathrm{F} \_$obs $\left.\right|^{2}$ is used in the Patterson function. All phases equal 0 . The value of the function is non-negative. The Patterson peaks are broad. The result of atoms and centrosymmetric cubic space groups is that an approximate structure of the crystal is contained in the peaks of the calculated electron densities [1]. We apply this property to the crystal of the Patterson function, to sharpen the Patterson peaks. We use a hydrogen atom with random coordinates in the general position of the space group of the crystal of the Patterson function. Combine the phases with all $\mid \mathrm{F} \_$obs $\left.\right|^{2}$. Some of these phases have signs -1 . Therefore, some electron densities are negative, and some Patterson peaks are sharpened. We apply this method to $\mathrm{Ba}_{3} \mathrm{Y}_{2} \mathrm{~B}_{6} \mathrm{O}_{15}$ [2]. Space group of the Patterson function is Im-3. Some results are presented in Table 1 and Fig.1.

Table 1. Some peaks in the sharpened Patterson function.

\begin{tabular}{llllll}
\hline Label & $x$ & $y$ & $z$ & Peak height & Half-width \\
\hline Origin & 0.000 & 0.000 & 0.000 & 14076 & $0.0361^{\mathrm{s}}$ \\
Ba1 - Y1 & 0.344 & 0.000 & 0.251 & 3545 & $0.0253^{\mathrm{s}}$ \\
Ba1 - O1 & 0.322 & 0.000 & -0.077 & $12074^{\mathrm{h}}$ & $0.0421^{\mathrm{s}}$ \\
Y1 - O1 & -0.049 & 0.000 & -0.321 & $14940^{\mathrm{h}}$ & 0.0517
\end{tabular}

${ }^{\mathrm{h}}$ higher peak height. ${ }^{\mathrm{s}}$ smaller half-width (Compare with peaks in the Patterson function)

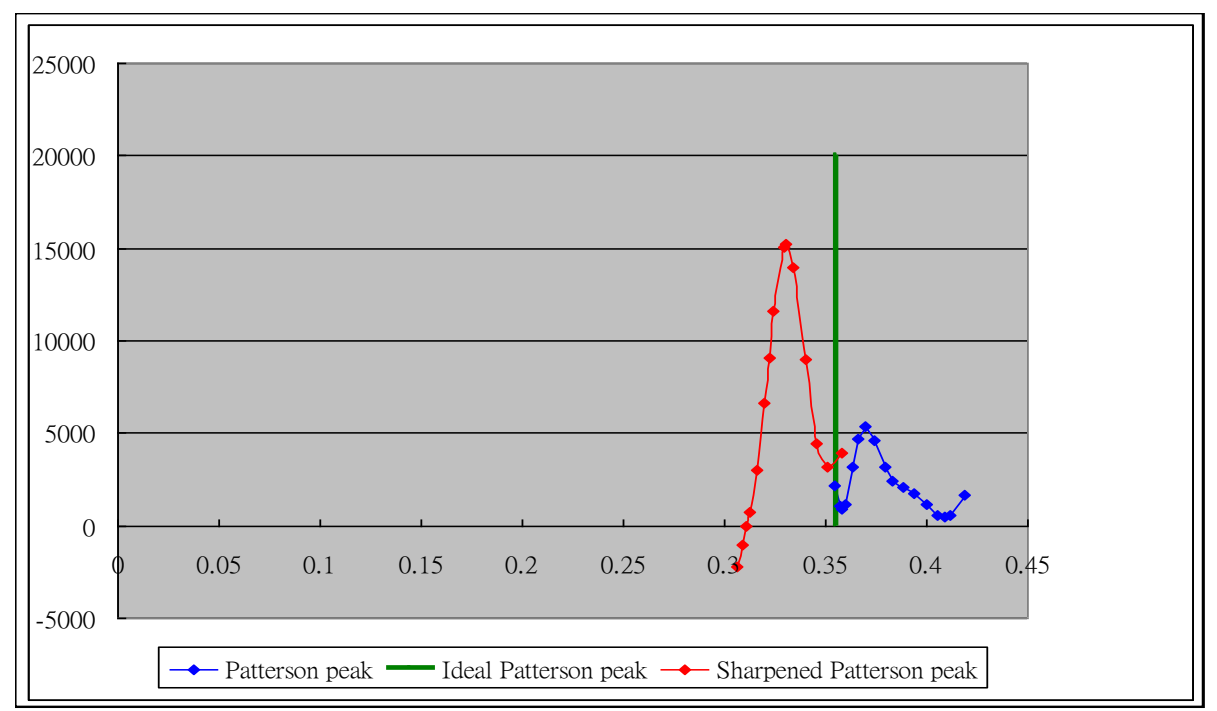

Figure 1. Sharpened Patterson peak Ba1-O1

[1] Yuen, P. S. Result of using atoms and centrosymmetric cubic space groups. (Unpublished).

[2] Zhao, S., Yao, J., Zhang, G., Fu, P. \& Wu, Y. (2011). Acta Cryst. C67, i39.

Keywords: atom; centrosymmetric cubic space group; sharpening.; 